\title{
Rationale and Design of a Study to Examine Lower Targets for Low-Density Lipoprotein-Cholesterol and Blood Pressure in Coronary Artery Disease Patients
} The Japanese Coronary Artery Disease Study II (JCADII)

\author{
Ryozo Nagai, MD; Tohru Izumi, MD*; Masahiko Kurabayashi, MD**; Hiroyuki Daida, MD ; \\ Taiki Tojo, MD*; Akira Hasegawa, MD**; Katsumi Miyauchi, MD ${ }^{\dagger}$; Dobun Hayashi, MD ${ }^{\dagger}$; \\ Takahide Kohro, MD $\dagger$; Yoshihiro Okada, MD $\pitchfork$; Tsutomu Yamazaki, MD \\ on behalf of the JCADII Investigators
}

\begin{abstract}
Background The benefits of coronary risk modification through medication and other methods have been shown in many clinical studies. Recently, aggressive lowering of low-density lipoprotein-cholesterol (LDL-C) has been shown to confer additive benefits in patients with coronary artery disease (CAD). However, it has not been shown in Japanese patients with CAD if multiple aggressive medical interventions for coronary risk factors are beneficial compared with standard regimens, so a prospective, randomized, open-label, blinded-endpoint (PROBE) multicenter study was designed to evaluate whether aggressive lowering of LDL-C and blood pressure in Japanese hypertensive, hypercholesterolemic CAD patients bestows additional benefits compared with regimens based on current Japanese guidelines.

Methods and Results Seventeen hospitals in Japan are participating in the Japanese Coronary Artery Disease II (JCADII) study. Hypertensive and hypercholesterolemic patients who have $\geq 75 \%$ stenosis in at least one major coronary artery according to American Heart Association guidelines will be allocated randomly to receive either conventional or aggressive therapy. Standard therapy for hypertension and hypercholesterolemia aims to reduce blood pressure to $<140 / 90 \mathrm{mmHg}$ and LDL-C concentration to $<100 \mathrm{mg} / \mathrm{dl}$. Aggressive therapy aims for targets of $<120 / 80 \mathrm{mmHg}$ and $<80 \mathrm{mg} / \mathrm{dl}$, respectively. We plan to recruit 500 patients and follow them up for 3 years. Antihypertensive agents, when used, include the angiotensin receptor blockers candesartan and/or losartan. Antihypercholesterolemic agents, when used, include at least one of the following statins: pravastatin, simvastatin, and atorvastatin.
\end{abstract}

Conclusion The JCADII study will provide important information concerning medical treatment of coronary risk factors in Japanese patients with CAD (UMIN-ID: UMIN000000571). (Circ J 2008; 72: 515-520)

Key Words: Aggressive therapy; Angiotensin receptor blockers; Coronary artery disease; PROBE study design; Statins

M any epidemiological studies, such as the Framingham study, have shown that risk factors for coronary artery disease (CAD) include hypertension, hyperlipidemia, obesity, smoking and diabetes mellitus. Many clinical studies have shown that medical and other forms of coronary risk intervention are effective

(Received July 19, 2007; revised manuscript received November 9 , 2007; accepted November 26, 2007)

Department of Cardiovascular Medicine, Graduate School of Medicine, The University of Tokyo, Tokyo, *Department of Cardioangiology, Kitasato University School of Medicine, Sagamihara, **Department of Medicine and Biological Science, Graduate School of Medicine, University of Gunma, Maebashi, Department of Cardiology, Juntendo University School of Medicine, tDepartment of Translational Research for Healthcare and Clinical Science and *Department of Clinical Epidemiology and Systems, Graduate School of Medicine, The University of Tokyo, Tokyo, Japan

This work was supported by a grant from the Japan Heart Foundation. Mailing address: Tsutomu Yamazaki, MD, Department of Clinical Epidemiology and Systems, Graduate School of Medicine, The University of Tokyo, 7-3-1 Hongo, Bunkyo-ku, Tokyo 113-8655, Japan All rights are reserved to the Japanese Circulation Society. For permissions, please e-mail: cj@j-circ.or.jp in reducing cardiovascular events in the settings of both primary prevention and secondary prevention-2-5 Therapeutic guidelines have been devised based on such knowledge, although it is now understood that ethnic differences affect risk factors and outcomes ${ }^{6-8}$

For a long period now, Japanese people have adopted Western lifestyles, resulting in their serum lipid concentrations becoming similar to those of Western people? However, the incidence rate of CAD remains unexpectedly low in Japanese people despite the increase in risk factors $!^{10}$ This kind of ethnic difference led us to conduct a large-scale, observational study of Japanese patients with CAD!1 ${ }^{11}$ The initial results of the study showed that, although the incidence rate of CAD in the general Japanese population might be lower, the incidence rate of secondary cardiovascular events in patients with CAD is similar to that in Western people! ${ }^{2}$

Recently, the results of several clinical studies showed the additive benefit of multiple medical interventions ${ }^{13}$ and aggressive lowering of low-density lipoprotein-cholesterol (LDL-C $)^{14-16}$ in reducing cardiovascular events. Yet, there have been no studies conducted to measure the effects of 
Table 1 Inclusion Criteria

1. Significant stenosis* in at least one major coronary artery

2. Aged between 20 and 79 years

3. Hyperlipidemia

4. Hypertension

5. Written informed consent obtained from the participant

*Significant stenosis of the coronary artery is defined as $\geq 75 \%$ stenosis, according to American Heart Association guidelines.

multiple and aggressive medical interventions for coronary risk factors in Japanese patients with CAD; hence, the Japanese Coronary Artery Disease II (JCADII) study was designed to evaluate the efficacy and safety of such regimens.

\section{Methods}

\section{Study Population}

Hypertensive and hypercholesterolemic patients of either gender with significant stenosis in at least one major coronary artery are eligible to participate. Significant stenosis of the coronary artery is defined as $\geq 75 \%$ stenosis according to American Heart Association guidelines ${ }^{17}$ Diagnosis can be made by either standard coronary angiography or coronary computed tomography angiogram.

The inclusion criteria are: between 20 and 79 years of age; hypercholesterolemia, defined as a serum total cholesterol concentration of $\geq 220 \mathrm{mg} / \mathrm{dl}$ in the past or during current therapy with statins and/or fibrates; hypertension, defined as systolic blood pressure (SBP) of $\geq 140 \mathrm{mmHg}$ and/or diastolic blood pressure (DBP) of $\geq 90 \mathrm{mmHg}$ in the past or during current therapy with antihypertensive agents; and the participant's written informed consent (Table 1).

Exclusion criteria are: known allergic reaction to the study drugs; familial hypercholesterolemia; uncontrolled hypercholesterolemia (serum LDL-C concentration remains $\geq 180 \mathrm{mg} / \mathrm{dl}$ despite conventional therapy); uncontrolled hypertension (SBP remains $\geq 180 \mathrm{mmHg}$ and/or DBP $\geq 110 \mathrm{mmHg}$ despite conventional therapy); myocardial infarction or stroke within the past 3 months; secondary hypertension; secondary hyperlipidemia (although diabetes mellitus-induced hyperlipidemia is not excluded); severe valvular disease; uncontrolled severe arrhythmia, such as recurrent ventricular fibrillation or ventricular tachycardia; renal dysfunction, defined as a serum creatinine concentration of $\geq 2.5 \mathrm{mg} / \mathrm{dl}$; hepatic dysfunction, defined as a concentration of alanine aminotransferase or aspartate aminotransferase 3 times the normal upper limit; pregnancy or possible pregnancy; and the decision by the physician in charge that the patient is unsuitable for inclusion (Table 2).

\section{Study Design}

The JCADII study is a multicenter, prospective, random-

Table 2 Exclusion Criteria

1. Known allergy to study drugs

2. Familial hypercholeterolemia

3. Uncontrolled severe hypercholesterolemia (low-density lipoprotein cholesterol $\geq 180 \mathrm{mg} / \mathrm{dl}$ )

4. Uncontrolled severe hypertension (systolic blood pressure $\geq 180 \mathrm{mmHg}$ and/or diastolic blood pressure $\geq 110 \mathrm{mmHg}$ )

5. Myocardial infarction or stroke $<3$ months before registration

6. Secondary hypertension

7. Secondary hyperlipidemia

8. Severe valvular disease

9. Uncontrolled severe arrhythmia

10. Renal dysfunction (defined as serum creatinine concentration $\geq 2.5 \mathrm{mg} / \mathrm{dl}$ )

11. Hepatic dysfunction (defined as an alanine aminotransferase or aspartate aminotransferase 3 times the normal upper limit)

12. Pregnancy or possible pregnancy

13. Decision by the attending physician that the patient is unsuitable for inclusion

\footnotetext{
Intensive therapy

- Therapeutic targets: SBP/DBP $<120 / 80 \mathrm{mmHg}$, LDL-C $<80 \mathrm{mg} / \mathrm{d}$

- ARBs: losartan, candesartan

- Statins: pravastatin, simvastatin, atorvastatin

- Additional drug(s)*

Run-in period

Conventional therapy

- Therapeutic targets: SBP/DBP < 140/90 mmHg, LDL-C < 100 mg/dl

- ARBs: losartan, candesartan

- Statins: pravastatin, simvastatin, atorvastatin

- Additional drug(s)*
}

Fig 1. Study design. *Any drug can be added except angiotensin receptor blockers not specified in the study, statins not specified in the study and angiotensin-converting enzyme inhibitors. DBP, diastolic blood pressure; LDLC, low-density lipoprotein-cholesterol; SBP, systolic blood pressure. 
Table 3 Schedule for Data Collection

\begin{tabular}{lccccccccc}
\hline \hline & \multirow{8}{*}{ Run-in period } & \multicolumn{7}{c}{ Study period (months) } \\
\cline { 3 - 8 } & & 0 & 3 & 6 & 12 & 18 & 24 & 30 & 36 \\
\hline Patient data & $M$ & & & & & & & \\
Blood pressure & $M$ & $M$ & $M$ & $M$ & $M$ & $M$ & $M$ & $M$ & $M$ \\
Serum lipid concentrations & $M$ & $M$ & $M$ & $M$ & $M$ & $M$ & $M$ & $M$ & $M$ \\
Pulse wave pressure & & $O$ & $O$ & $O$ & $O$ & & $O$ & $O$ \\
Echocardiography & & $M$ & $O$ & $O$ & $M$ & $M$ & $M$ \\
Other blood tests & $M$ & $M$ & $M$ & $M$ & & $M$ & $M$ \\
Urinary tests & & $M$ & $M$ & $M$ & $M$ & & $M$ & $M$ \\
\hline
\end{tabular}

O, optional; $M$, mandatory.

ized, open-label, blinded-endpoint (PROBE) trial carried out at 17 cardiology centers in Japan. The first patient was enrolled in November 2006, and randomization is expected to end in October 2007. All eligible patients will be allocated randomly to either conventional therapy or aggressive therapy. Randomization is being performed on the basis of the following baseline variables: SBP, serum LDL-C concentration, diabetes mellitus and congestive heart failure. Fig 1 shows the study design.

The therapeutic targets for patients assigned to conventional therapy are an SBP of $<140 \mathrm{mmHg}$ and a DBP of $<90 \mathrm{mmHg}$ for hypertension, and a serum LDL-C concentration of $<100 \mathrm{mg} / \mathrm{dl}$ for hypercholesterolemia. If the patient has diabetes mellitus, the therapeutic targets for hypertension are an SBP of $<130 \mathrm{mmHg}$ and a DBP of $<80 \mathrm{mmHg}$, according to current Japanese guidelines for hypertension therapy 18 The therapeutic targets for patients assigned to intensive therapy are an SBP of $<120 \mathrm{mmHg}$ and a DBP of $<80 \mathrm{mmHg}$ for hypertension, and a serum LDL-C concentration of $<80 \mathrm{mg} / \mathrm{dl}$ for hypercholesterolemia.

Antihypertensive therapy should include the angiotensin receptor blockers candesartan and/or losartan. Antihypercholesterolemia therapy should include at least one of the following statins: pravastatin, simvastatin and atorvastatin. A no drug regimen is allowed if the targets are met with nonpharmacological therapy. Additional therapy can be used to achieve the target blood pressure and LDL-C concentration, except for angiotensin receptor blockers not specified above, statins not specified above and angiotensin-converting enzyme inhibitors. It is expected that the therapeutic targets for each patient will be achieved within 3 months of randomization.

The schedule for data collection is shown in Table 3. Patient data include the date on which consent was obtained, date of birth, gender, height, weight, history of cardiovascular disease, comorbid diseases, history of smoking, waist circumference, and year and method of coronary angiogram. Blood pressure measurements include both SBP and DBP. Serum lipid concentrations include high-density lipoprotein-cholesterol, triglycerides and LDL-C, where LDL$\mathrm{C}$ is measured by homogeneous assays. Measurement of total cholesterol is not mandatory but data registration is possible and the registered data will be used for later investigations. The measured values of LDL-C are normalized using standard samples sent to each institute. Homogeneous assays of LDL-C measurement were assumed not to vary significantly from the Friedewald calculation method based on studies that showed that LDL-C values obtained by the Friedewald method and homogeneous methods correlate well with each other, in general19,20 Pulse wave pressure is measured at facilities where it is available. Interventricular septum thickness and ejection fraction are measured by echocardiography. Other blood tests include measurement of white blood cell count, hemoglobin, platelet count, fasting blood glucose, hemoglobin A1c protein (for diabetic patients only), alanine aminotransferase, glutamyl transpeptidase, blood urea nitrogen, uric acid, serum creatinine, creatine kinase, high-sensitive C-reactive protein and Btype natriuretic peptide. Urinary tests include measurements of urinary glucose and protein. It is expected that all patients are followed up for 3 years.

\section{Study Management}

The study was initiated by the investigators and is scientifically led by a steering committee consisting of independent researchers and investigators. This committee has full responsibility for the study protocol and publications arising from the study. The committee is granted full access to all data at the study's completion. Data registration is performed through a Web-based interface by each investigator or clinical research coordinator at each facility. All data will be collected in a central data server. An independent monitoring board, which is not involved in the conduct of the trial, will monitor aggregated endpoint summaries and medically serious adverse events on an ongoing basis.

\section{Endpoints}

The primary endpoint is a composite of all deaths, nonfatal myocardial infarction, unstable angina pectoris, coronary artery bypass graft operation, non-fatal stroke, non-fatal major vascular diseases and peripheral artery diseases. Secondary endpoints are the occurrence of any primary event; all-cause hospitalization; hospitalization due to congestive heart failure; percutaneous coronary intervention; transient ischemic attack; blood pressure; pulse wave velocity; concentrations of serum lipid, high-sensitive C-reactive protein and B-type natriuretic peptide; left ventricular hypertrophy; and safety.

\section{Sample Size}

Sample size calculation is based on the Steno- 2 study 13 and the JCAD study $!^{2}$ Steno-2 suggested that at least a $41 \%$ risk reduction in cardiovascular events would be achieved in the aggressive therapy group compared with the conventional therapy group. The JCAD study showed that the annual event rate in Japanese patients with CAD is approximately $6 \%$. The MEGA study showed that a moderate $23 \mathrm{mg} / \mathrm{dl}$ lowering of LDL-C resulted in 33\% reduction of CAD in a low-risk Japanese population. These findings, combined with a detection power of $80 \%$ and a 2 -sided significance level of 5\%, enabled us to calculate that the number of patients needed to detect a significant difference between the 
2 groups in 3 years is 244 for each group. Assuming that the dropout rate is $2 \%$, the number of patients needed is 250 for each group.

\section{Analysis}

When the study is completed, the primary and secondary endpoints will be analyzed using survival analysis techniques on the basis of time to first event. A comparison of the results for the aggressive therapy group with those for the conventional therapy group will be done with KaplanMeier curves and a log-rank test. The non-adjusted hazard ratio of each variable will be calculated using a univariate Cox hazards model. The adjusted hazards ratio of each variable will be calculated using a multivariate Cox hazards model.

Interim analyses will be performed every year after the initiation of the study to evaluate the primary efficacy endpoint for major cardiovascular events and safety. The independent monitoring board may advise early termination of the trial for safety, scientific or ethical reasons.

\section{Ethical Considerations}

The Institutional Review Board of each participating institution reviewed and approved the study protocol and other documents. As for informed consent, each attending physician explained the study to each candidate patient, who gave voluntary written informed consent prior to enrolment.

\section{Role of Funding Source}

The sponsor of the study has no role in the study design, data collection, data analysis, data interpretation or publication of any articles arising from the study.

\section{Discussion}

The incidence rate of CAD in the general Japanese population remains low ${ }^{10}$ despite adopting Westernized lifestyles, having similar serum lipid concentrations to those of Western people, and high smoking rates ${ }^{21}$ Nonetheless, we have shown through analysis of data from a large cohort of CAD patients diagnosed using coronary angiography that the rate of secondary cardiovascular events in Japanese patients with CAD is similar to that in Western people! ${ }^{12}$

Epidemiological studies, such as the Framingham study, established coronary risk factors, such as hypertension, hyperlipidemia, smoking, aging and being male! Many clinical studies have shown that medical interventions to modify coronary risk factors are effective in reducing cardiovascular events in settings of both primary prevention and secondary prevention ${ }^{2-5,22,23}$

Therapeutic guidelines have been devised according to the accumulated evidence and are being used in clinical settings. Cardiovascular events increase as the number and extent of risk factors increase. This knowledge led to several clinical studies that evaluated the efficacy and safety of multiple medical interventions for cardiovascular risk factors ${ }^{13}$ or aggressive lowering of LDL-C,14-16,24 showing that these approaches confer additive benefits in reducing cardiovascular events.

To the best of our knowledge, there have not been any studies that evaluated the effects of multiple aggressive coronary risk interventions in Japanese patients with CAD. Therefore, the JCADII study was designed to assess the efficacy and safety of aggressive lowering of blood pressure and
LDL-C in patients who have hypertension, hyperlipidemia, and CAD confirmed angiographically or by computed tomography.

As mentioned earlier, several clinical studies have shown that aggressive lowering of LDL-C is effective in the settings of both primary prevention of cardiovascular events 5 and secondary prevention of cardiovascular events, ${ }^{4}$ be it in the stable phase ${ }^{15}$ or in the post-acute event phase ${ }^{14}$ As for evidence in a Japanese population, the only randomized control study on lipid-lowering therapy in Japanese showed that a moderate decrease in LDL-C $(23 \mathrm{mg} / \mathrm{dl})$ resulted in a substantial decrease of CAD events. ${ }^{25}$ An observational study conducted in Japan showed that lowering the LDL-C level below $100 \mathrm{mg} / \mathrm{dl}$ in hypercholesterolemic patients is safe and effective in reducing coronary events ${ }^{26}$ Additionally, aggressive statin therapy was shown to confer beneficial effects on coronary atherosclerotic lesions compared to usual lipid-lowering therapies ${ }^{27}$ These studies suggest that aggressive lowering of LDL-C is safe and might be beneficial in reducing cardiovascular events in the Japanese population.

As for blood pressure lowering, there has long been a debate about the so-called J-curve phenomenon? ${ }^{2-30}$ Theoretically, it can be argued that lowering blood pressure too much, especially DBP, might result in impaired coronary flow, which might reduce even further the coronary flow of CAD patients and result in cardiovascular events. The results of the Hypertension Optimal Treatment study showed that a target DBP of $<80 \mathrm{mmHg}$ is safe, and no J-curve effect was observed in the primary prevention of cardiovascular events in hypertensive patients? The results of the Antihypertensive and Lipid-lowering Treatment to Prevent Heart Attack trial also showed that achieving an average DBP of $<80 \mathrm{mmHg}$ is tolerated well and is effective in reducing major cardiovascular events in high-risk hypertensive patients? ${ }^{3}$ However, not all the patients included in these trials had been rigorously defined as having CAD.

Recently, a subanalysis of the International VerapamilTrandolapril Study, which was conducted on patients with documented CAD, showed that when the study's participants were categorized into 7 strata according to blood pressure, a J-curve phenomenon was observed 32 However, this result was obtained by post hoc analysis, and, although the background profiles of patients were taken into account when performing statistical analyses, it is not clear whether the worse profile of the lowest blood pressure strata was accounted for appropriately. Although there is scarce direct evidence regarding what level of blood pressure should be attained by medication in elderly Japanese hypertensive patients, an observational study has reported that those in the general population with optimal blood pressure $(<120 /$ $<80 \mathrm{mmHg}$ ) have the lowest risk for cardiovascular disease $3^{3}$ Recently, chronic kidney disease is reported to be an independent risk factor for cardiovascular events 34 An observational study conducted in Japan showed that when blood pressure was categorized into 6 levels, those with optimal blood pressure $(<120 /<80 \mathrm{mmHg})$ had the lowest risk for developing end stage renal disease 35 suggesting the benefits of lowering blood pressure to this level. To date, there have been no clinical trials that show directly whether aggressively lowering blood pressure in CAD patients is safe and effective in reducing cardiovascular events, and such a study is warranted.

In conclusion, the JCADII study is the first to evaluate whether aggressive lowering of LDL-C and blood pressure 
is safe and effective in reducing cardiovascular events in hypertensive, hypercholesterolemic Japanese patients with $\mathrm{CAD}$. The results will add important information regarding the treatment of such patients.

\section{References}

1. Lerner DJ, Kannel WB. Patterns of coronary heart disease morbidity and mortality in the sexes: A 26-year follow-up of the Framingham population. Am Heart J 1986; 111: 383-390.

2. Hansson L, Zanchetti A, Carruthers SG, Dahlof B, Elmfeldt D, Julius $\mathrm{S}$, et al. Effects of intensive blood-pressure lowering and low-dose aspirin in patients with hypertension: Principal results of the Hypertension Optimal Treatment (HOT) randomised trial: HOT Study Group. Lancet 1998; 351: 1755-1762.

3. Pepine CJ, Handberg EM, Cooper-DeHoff RM, Marks RG, Kowey P, Messerli FH, et al; INVEST Investigators. A calcium antagonist vs a non-calcium antagonist hypertension treatment strategy for patients with coronary artery disease: The International Verapamil-Trandolapril Study (INVEST): A randomized controlled trial. JAMA 2003; 290: $2805-2816$.

4. Randomised trial of cholesterol lowering in 4444 patients with coronary heart disease: The Scandinavian Simvastatin Survival Study (4S). Lancet 1994; 344: 1383-1389.

5. Shepherd J, Cobbe SM, Ford I, Isles CG, Lorimer AR, MacFarlane PW, et al. Prevention of coronary heart disease with pravastatin in men with hypercholesterolemia: West of Scotland Coronary Prevention Study Group. N Engl J Med 1995; 333: 1301-1307.

6. Pristipino C, Beltrame JF, Finocchiaro ML, Hattori R, Fujita M, Mongiardo R, et al. Major racial differences in coronary constrictor response between Japanese and Caucasians with recent myocardial infarction. Circulation 2000; 101: 1102-1108.

7. Sekikawa A, Satoh T, Hayakawa T, Ueshima H, Kuller LH. Coronary heart disease mortality among men aged 35-44 years by prefecture in Japan in 1995-1999 compared with that among white men aged 35-44 by state in the United States in 1995-1998: Vital statistics data in recent birth cohort. Jpn Circ J 2001; 65: 887-892.

8. Verschuren WM, Jacobs DR, Bloemberg BP, Kromhout D, Menotti A, Aravanis $\mathrm{C}$, et al. Serum total cholesterol and long-term coronary heart disease mortality in different cultures: Twenty-five-year followup of the seven countries study. JAMA 1995; 274: 131-136.

9. Egusa G, Yamane K. Lifestyle, serum lipids and coronary artery disease: Comparison of Japan with the United States. J Atheroscler Thromb 2004; 11: 304-312.

10. van den Hoogen PC, Feskens EJ, Nagelkerke NJ, Menotti A, Nissinen A, Kromhout D. The relation between blood pressure and mortality due to coronary heart disease among men in different parts of the world: Seven Countries Study Research Group. N Engl J Med 2000; 342: $1-8$.

11. Hayashi D, Yamazaki T. Design and rationale of the Japanese Coronary Artery Disease (JCAD) Study: A large-scale, multicentered prospective cohort study. Jpn Heart J 2004; 45: 895-911.

12. The Japanese Coronary Artery Disease (JCAD) Study Investigators. Current status of the background of patients with coronary artery disease in Japan: The Japanese Coronary Artery Disease Study (The JCAD Study). Circ J 2006; 70: 1256-1262.

13. Gaede P, Vedel P, Larsen N, Jensen GV, Parving HH, Pedersen O. Multifactorial intervention and cardiovascular disease in patients with type 2 diabetes. $N$ Engl J Med 2003; 348: 383-393.

14. Cannon CP, Braunwald E, McCabe CH, Rader DJ, Rouleau JL, Belder R, et al. Intensive versus moderate lipid lowering with statins after acute coronary syndromes. N Engl J Med 2004; 350: $1495-$ 1504.

15. LaRosa JC, Grundy SM, Waters DD, Shear C, Barter P, Fruchart JC, et al. Intensive lipid lowering with atorvastatin in patients with stable coronary disease. $N$ Engl J Med 2005; 352: 1425-1435.

16. Pedersen TR, Faergeman O, Kastelein JJ, Olsson AG, Tikkanen MJ, Holme I, et al. High-dose atorvastatin vs usual-dose simvastatin for secondary prevention after myocardial infarction: The IDEAL study: A randomized controlled trial. JAMA 2005; 294: 2437-2445.

17. Austen WG, Edwards JE, Frye RL, Gensini GG, Gott VL, Griffith LS, et al. A reporting system on patients evaluated for coronary artery disease: Report of the Ad Hoc Committee for Grading of Coronary Artery Disease, Council on Cardiovascular Surgery, American Heart Association. Circulation 1975; 51: 5-40.

18. Rakugi H, Ogihara T. Guidelines for treatment of hypertension: JSH2004. Nippon Ronen Igakkai Zasshi 2006; 43: 569-573 (in Japanese).

19. Teerakanchana T, Puavilai W, Suriyaprom K, Tungtrongchitr R.
Comparative study of LDL-cholesterol levels in Thai patients by the direct method and using the Friedewald formula. Southeast Asian J Trop Med Public Health 2007; 38: 519-527.

20. Cordova CM, Schneider CR, Juttel ID, Cordova MM. Comparison of LDL-cholesterol direct measurement with the estimate using the Friedewald formula in a sample of 10,664 patients. Arq Bras Cardiol 2004; 83: 482 - 487; 476-481.

21. Sekikawa A, Ueshima H, Kadowaki T, El-Saed A, Okamura T, Takamiya T, et al. Less subclinical atherosclerosis in Japanese men in Japan than in White men in the United States in the post-World War II birth cohort. Am J Epidemiol 2007; 165: 617-624.

22. Dormandy JA, Charbonnel B, Eckland DJ, Erdmann E, MassiBenedetti M, Moules IK, et al. Secondary prevention of macrovascular events in patients with type 2 diabetes in the PROactive Study (PROspective pioglitAzone Clinical Trial In macroVascular Events): A randomised controlled trial. Lancet 2005; 366: 1279-1289.

23. Tight blood pressure control and risk of macrovascular and microvascular complications in type 2 diabetes: UKPDS 38: UK Prospective Diabetes Study Group. BMJ 1998; 317: 703-713.

24. Sever PS, Dahlof B, Poulter NR, Wedel H, Beevers G, Caulfield M, et al. Prevention of coronary and stroke events with atorvastatin in hypertensive patients who have average or lower-than-average cholesterol concentrations, in the Anglo-Scandinavian Cardiac Outcomes Trial-Lipid Lowering Arm (ASCOT-LLA): A multicentre randomised controlled trial. Lancet 2003; 361: 1149-1158.

25. Nakamura H, Arakawa K, Itakura H, Kitabatake A, Goto Y, Toyota $\mathrm{T}$, et al. Primary prevention of cardiovascular disease with pravastatin in Japan (MEGA Study): A prospective randomised controlled trial. Lancet 2006; 368: 1155-1163.

26. Mabuchi H, Kita T, Matsuzaki M, Matsuzawa Y, Nakaya N, Oikawa $\mathrm{S}$, et al. Large scale cohort study of the relationship between serum cholesterol concentration and coronary events with low-dose simvastatin therapy in Japanese patients with hypercholesterolemia and coronary heart disease: Secondary prevention cohort study of the Japan Lipid Intervention Trial (J-LIT). Circ J 2002; 66: 1096-1100.

27. Okazaki S, Yokoyama T, Miyauchi K, Shimada K, Kurata T, Sato H, et al. Early statin treatment in patients with acute coronary syndrome: Demonstration of the beneficial effect on atherosclerotic lesions by serial volumetric intravascular ultrasound analysis during half a year after coronary event: The ESTABLISH Study. Circulation 2004; 110: $1061-1068$.

28. Cruickshank JM, Thorp JM, Zacharias FJ. Benefits and potential harm of lowering high blood pressure. Lancet 1987; 1: 581 -584.

29. Cruickshank J. The J-curve in hypertension. Curr Cardiol Rep 2003; 5: $441-452$.

30. Hansson L. How far should blood pressure be lowered?: What is the role of the J-curve? Am J Hypertens 1990; 3: 726-729; Discussion $730-732$.

31. Major cardiovascular events in hypertensive patients randomized to doxazosin vs chlorthalidone: The antihypertensive and lipid-lowering treatment to prevent heart attack trial (ALLHAT): ALLHAT Collaborative Research Group. JAMA 2000; 283: 1967-1975.

32. Messerli FH, Mancia G, Conti CR, Hewkin AC, Kupfer S, Champion A, et al. Dogma disputed: Can aggressively lowering blood pressure in hypertensive patients with coronary artery disease be dangerous? Ann Intern Med 2006; 144: 884-893.

33. Arima H, Tanizaki Y, Kiyohara Y, Tsuchihashi T, Kato I, Kubo M, et al. Validity of the JNC VI recommendations for the management of hypertension in a general population of Japanese elderly: The Hisayama study. Arch Intern Med 2003; 163: 361-366.

34. Go AS, Chertow GM, Fan D, McCulloch CE, Hsu CY. Chronic kidney disease and the risks of death, cardiovascular events, and hospitalization. N Engl J Med 2004; 351: 1296-1305.

35. Tozawa M, Iseki K, Iseki C, Kinjo K, Ikemiya Y, Takishita S. Blood pressure predicts risk of developing end-stage renal disease in men and women. Hypertension 2003; 41: 1341-1345.

\section{Appendix 1}

Steering Committee: Ryozo Nagai (Chief, Department of Cardiovascular Medicine, Graduate School of Medicine, The University of Tokyo, Tokyo); Tsutomu Yamazaki (Department of Clinical Epidemiology and Systems, Graduate School of Medicine, Faculty of Medicine, The University of Tokyo, Tokyo); Masahiko Kurabayashi (Department of Medicine and Biological Science, Graduate School of Medicine, University of Gunma, Maebashi); Tohru Izumi (Department of Cardioangiology, Kitasato University School of Medicine, Sagamihara); Hiroyuki Daida (Department of Cardiology, Juntendo University School of Medicine, Tokyo).

Protocol Committee: Akira Hasegawa (Department of Medicine and Biological Science, Graduate School of Medicine, University of Gunma, 
Maebashi); Taiki Tojo (Department of Cardioangiology, Kitasato University School of Medicine, Sagamihara); Katsumi Miyauchi (Department of Cardiology, Juntendo University School of Medicine, Tokyo); Tsutomu Yamazaki (Department of Clinical Epidemiology and Systems, Graduate School of Medicine, Faculty of Medicine, The University of Tokyo, Tokyo); Dobun Hayashi, Takahide Kohro and Yoshihiro Okada (Department of Translational Research for Healthcare and Clinical Science, Graduate School of Medicine, The University of Tokyo, Tokyo).

Data Management and Statistics: Tsutomu Yamazaki, Hiroshi Ohtsu, and Shinya Suzuki (Department of Cardiovascular Medicine, Graduate School of Medicine, The University of Tokyo, Tokyo).

Safety Committee: Dobun Hayashi, Takahide Kohro and Yoshihiro Okada (Department of Translational Research for Healthcare and Clinical Science, Graduate School of Medicine, The University of Tokyo, Tokyo); Yasushi Imai (Department of Cardiovascular Medicine, Graduate School of Medicine, The University of Tokyo, Tokyo).

Endpoint Committee: Sugao Ishiwata (Cardiovascular Center, Toranomon Hospital, Tokyo); Norio Tanahashi (Department of Neurology, Saitama Medical University, Saitama); Hiroshi Nonogi (Division of Cardiology, National Cardiovascular Center, Suita).

Independent Monitoring Board: Masatoshi Kawana (Department of Cardiology, Aoyama Hospital, Tokyo Women's Medical University, Tokyo); Hideo Mitamura (Department of Cardiology, Saiseikai Central Hospital, Tokyo); Morimasa Takayama (First Department of Internal Medicine, Nippon Medical School, Tokyo).

Members: Akihiko Nakano (Department of Medicine and Biological
Science, Graduate School of Medicine, University of Gunma, Maebashi); Norio Kanesawa and Toyoshi Sasaki (Department of Cardiology, National Hospital Organization Takasaki Hospital, Takasaki); Shigeru Ohshima, Hiroshi Hoshizaki and Takuji Toyama (Division of Cardiology, Gunma Prefectural Cardiovascular Center, Maebashi); Shuichi Ichikawa and Toshiya Iwasaki (Department of Internal Medicine, Cardiovascular Hospital of Central Japan, Gunma); Takesatoru Fukuda (Division of Cardiology, Saiseikai Maebashi Hospital, Maebashi); Toshiro Kurosawa and Takeshi Sasaki (Department of Cardiology, Machida Municipal Hospital, Tokyo); Hiroshi Ito and Daisuke Sato (Division of Cardiology, Numazu Municipal Hospital, Numazu); Takeo Kawaguchi, Ichiro Takeuchi and Masaru Yuge (Division of Cardiology, Odawara Municipal Hospital, Odawara); Koichiro Aihara (Department of Cardiology, Juntendo University School of Medicine, Tokyo); Yasumasa Fujiwara, Kenji Inoue and Shinya Okazaki (Department of Cardiology, Juntendo University Nerima Hospital, Tokyo); Tatsuji Kanoh, Yuji Nakazato and Kosei Tanimoto (Department of Cardiology, Juntendo University Urayasu Hospital, Urayasu); Masataka Sumiyoshi and Satoru Suwa (Department of Cardiology, Juntendo University Shizuoka Hospital, Izunokuni); Yasunobu Hirata (Department of Cardiovascular Medicine, Graduate School of Medicine, The University of Tokyo, Tokyo); Iwao Ishibashi (Department of Cardiology, Chiba Emergency Medical Center, Chiba); Akira Miyazaki and Yoshitake Nakamura (Department of Cardiology, Chiba Cardiovascular Center, Ichihara); Nobuyuki Komiyama (Department of Cardiology, Saitama Medical School, Saitama). 